\title{
Lattice QCD study of a five-quark hadronic molecule
}

\author{
M.S. Cook $^{1,2}$ and H.R. Fiebig ${ }^{1}$ \\ ${ }^{1}$ Physics Department, FIU-University Park, Miami, Florida 33199 \\ ${ }^{2}$ Department of Physics, Converse College, Spartanburg, South Carolina 29302
}

(Dated: October 30, 2018)

\begin{abstract}
We compute the ground-state energies of a heavy-light $K-\Lambda$ like system as a function of the relative distance $r$ of the hadrons. The heavy quarks, one in each hadron, are treated as static. Then, the energies give rise to an adiabatic potential $V_{a}(r)$ which we use to study the structure of the five-quark system. The simulation is based on an anisotropic and asymmetric lattice with Wilson fermions. Energies are extracted from spectral density functions obtained with the maximum entropy method. Our results are meant to give qualitative insight: Using the resulting adiabatic potential in a Schrödinger equation produces bound state wave functions which indicate that the ground state of the five-quark system resembles a hadronic molecule, whereas the first excited state, having a very small rms radius, is probably better described as a five-quark cluster, or a pentaquark. We hypothesize that an all light-quark pentaquark may not exist, but in the heavy-quark sector it might, albeit only as an excited state.
\end{abstract}

PACS numbers: 12.38.Gc, 12.39.Mk

\section{INTRODUCTION}

Lattice QCD studies of hadron-hadron interactions are the gateway to nuclear physics through first principles [1]. From a lattice simulation point of view the nucleonnucleon interaction is unquestionably the most challenging case 2], and it might not be resolved in the foreseeable future. However, interactions in other two-hadron systems are worth investigating as well, because new insights into the structural features of already discovered [3] or yet unknown baryon resonances may emerge. In particular, one may ask if some of those may be understood as hadronic molecules, like the deuteron, or if more compact clusters, like a pentaquark [4], may also exist.

We are here interested in pairs of hadrons containing one heavy quark each. In such systems a relative, residual, interaction is a well-defined concept. In the spirit of the Born-Oppenheimer approximation, the (slow) heavy quarks serve to define the centers of the two hadrons while the (fast) light quarks and gluons provide the physics of the interaction. Exploratory studies along those lines have been done in the context of meson-meson and baryon-baryon systems [5, [6, 7].

Specifically, we here investigate a heavy-light mesonbaryon (five-quark) hadron with the quantum numbers of an S-wave $K-\Lambda$ system. The heavy-quark propagator is treated in the static approximation. This allows us to compute the total energy as a function of the relative distance $r$ between the heavy quarks, viz. hadrons. A production scheme is illustrated in Fig. 1. The resulting adiabatic (Born-Oppenheimer) potential $V_{a}(r)$ then may be used to address the possibility of molecule-like, or other, structures.

In the static approximation to the heavy-quark propagator the potential $V_{a}(r)$ extracted from the lattice equally applies to the systems $K-\Lambda, D-\Lambda_{c}$, and $B-\Lambda_{b}$,

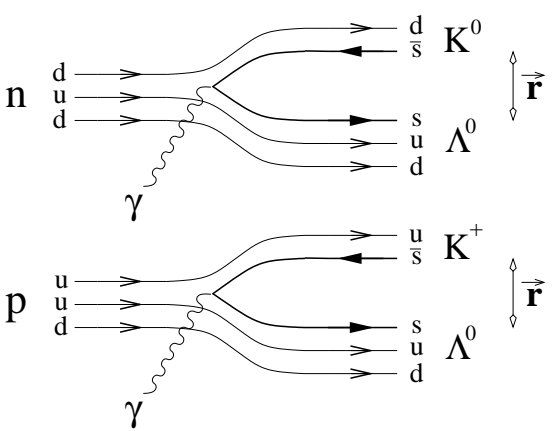

FIG. 1: Schematic view of $K-\Lambda$ like molecule production and illustration of the current scheme for extracting a hadronic interaction at relative distance $\vec{r}$. Thick lines indicate heavyquark propagators, and thin lines depict light quark propagators.

the $s$-flavored quarks being replaced with $c$ and $b$ flavors, respectively. Within the framework of a Schrödinger equation those systems can be studied using their respective physical reduced masses, provided of course, one is willing to accept the limitations of the adiabatic approximation and the non-relativistic nature of the framework. This is the main reason for classifying our results as qualitative.

\section{OPERATOR CONSTRUCTION}

The lattice simulation requires two-hadron interpolating fields. Those are constructed using as building blocks standard local operators for the $K^{+}$and $\Lambda^{0}$ particles [8]. They are placed at relative distance $\vec{r}$ and then projected to total momentum zero

$$
\mathcal{O}_{\alpha}(\vec{r} ; t)=N^{-1 / 2} \sum_{\vec{x}} \sum_{\vec{y}} \delta_{\vec{r}, \vec{x}-\vec{y}} K^{+}(\vec{x} t) \Lambda_{\alpha}^{0}(\vec{y} t) .
$$


Here the normalization $N$ is the spatial lattice volume, the sums are over lattice sites, and $\alpha$ is a Dirac spinor index. Then, with

$$
\overline{\mathcal{O}}_{\mu}(\vec{r} ; t)=\mathcal{O}_{\alpha}^{\dagger}(\vec{r} ; t) \gamma_{4, \alpha \mu}
$$

the time correlation function

$$
C\left(t, t_{0}\right)=\left\langle\mathcal{O}_{\mu}(\vec{r} ; t) \overline{\mathcal{O}}_{\mu}\left(\vec{s} ; t_{0}\right)\right\rangle-\left\langle\mathcal{O}_{\mu}(\vec{r} ; t)\right\rangle\left\langle\overline{\mathcal{O}}_{\mu}\left(\vec{s} ; t_{0}\right)\right\rangle,
$$

where $\vec{s}$ is the relative distance at the source, can be expressed in terms of fermion propagators. The flavor assignment $K^{+} \Lambda^{0} \sim \bar{s} u$ uds causes the separable term in (3) to vanish. Writing $H\left(\vec{x} t, \vec{y} t_{0}\right)$ and $G\left(\vec{x} t, \vec{y} t_{0}\right)$ for the heavy $(\mathrm{s})$ and light $(\mathrm{u}, \mathrm{d})$ quark propagators, respectively, one obtains

$$
\begin{aligned}
C\left(t, t_{0}\right)= & \left\langle\sum _ { \vec { y } } \left[ H(\vec{y} t, \vec{y}+\vec{r} t) H\left(\vec{r}_{1}+\vec{s} t_{0}, \vec{r}_{1} t_{0}\right)\right.\right. \\
& \left.-H\left(\vec{y} t, \vec{r}_{1} t_{0}\right) H\left(\vec{r}_{1}+\vec{s} t_{0}, \vec{y}+\vec{r} t\right)\right] \times \\
& G\left(\vec{y} t, \vec{r}_{1} t_{0}\right)\left[G\left(\vec{y} t, \vec{r}_{1} t_{0}\right) G\left(\vec{y}+\vec{r} t, \vec{r}_{1}+\vec{s} t_{0}\right)\right. \\
- & \left.\left.G\left(\vec{y} t, \vec{r}_{1}+\vec{s} t_{0}\right) G\left(\vec{y}+\vec{r} t, \vec{r}_{1} t_{0}\right)\right]\right\rangle .
\end{aligned}
$$

For clarity the rather involved color and spin index structure is not shown in (4). Also, translational invariance of the gauge field average \langle\rangle has been used to arrive at the above expression, and utilizing this freedom, an arbitrary space site $\vec{r}_{1}$ was introduced to fix the source locations. A diagrammatic representation of (44) is shown in Fig. 2
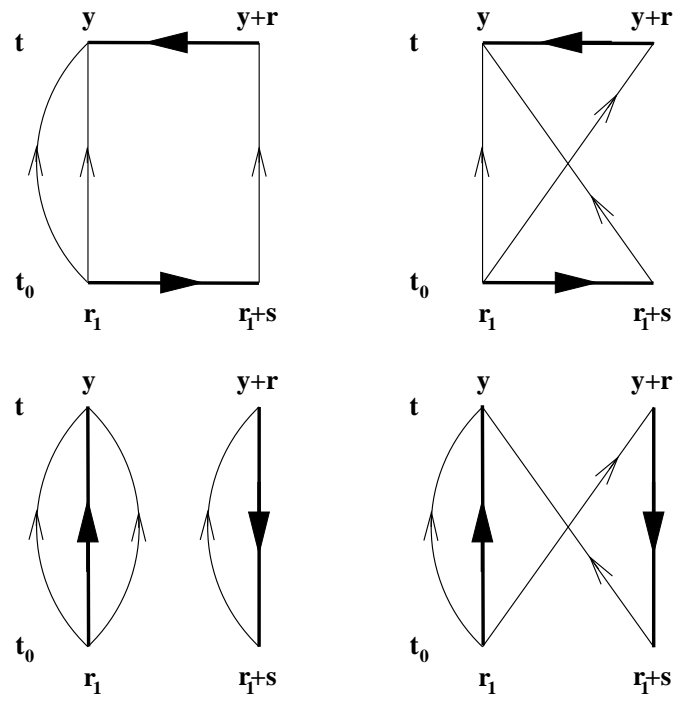

FIG. 2: Diagrammatic representation of (4). Thick lines indicate heavy-quark propagators, and thin lines depict light $(\mathrm{u}$ and d) quark propagators.

The heavy-quark propagators are employed in the static approximation. For (unimproved) Wilson fermions with hopping parameter $\kappa$ this means that the propagator is taken in the limit $\kappa \rightarrow 0$, resulting in

$$
H\left(\vec{x} t, \vec{y} t_{0}\right)=\delta_{\vec{x}, \vec{y}}(2 \kappa)^{t-t_{0}} \frac{1}{2}\left(1+\gamma_{4}\right) \mathcal{U}^{\dagger}\left(\vec{x} ; t_{0} t\right),
$$

where $\mathcal{U}\left(\vec{x} ; t_{0} t\right)$ is the product of $S U(3)$ link variables along a straight line from $\left(\vec{x} t_{0}\right)$ to $(\vec{x} t)[8]$. The factor $(2 \kappa)^{t-t_{0}}$ gives rise to only a constant global energy shift $\Delta m=-\ln (2 \kappa)$, which we will ignore.

The distance $\vec{r}=0$ is rather special [6] because a color singlet operator, as realized by (11), can also be achieved by a "color twisted" version of (1) where quarks across the hadrons $K^{+}$and $\Lambda^{0}$, now at the same location, are combined into a color singlet. Because we do not consider color twisted operators in this work, we restrict ourselves to non-zero relative distance[26]. Thus, using $H(\vec{y} t, \vec{y}+\vec{r} t) \propto \delta_{\vec{r}, \overrightarrow{0}}$ and $H\left(\vec{r}_{1}+\vec{s}_{0}, \vec{r}_{1} t_{0}\right) \propto \delta_{\vec{s}, \overrightarrow{0}}$, the first two terms in (4), and accordingly the top two diagrams in Fig. 2 vanish, and only the last two of those make a contribution to the correlation function for non-zero relative distance. By way of (5) those contributions are proportional to $\delta_{\vec{y}, \vec{r}_{1}} \delta_{\vec{r}, \vec{s}}$. Introducing $\vec{r}_{2}=\vec{r}_{1}+\vec{s}$, to replace $\vec{s}$, the correlation function (4) becomes

$$
\begin{aligned}
& C\left(t, t_{0}\right)=\delta_{\vec{r}, \vec{r}_{2}-\vec{r}_{1}}\left\langle H\left(\vec{r}_{1} t, \vec{r}_{1} t_{0}\right) H\left(\vec{r}_{2} t_{0}, \vec{r}_{2} t\right)\right. \\
& G\left(\vec{r}_{1} t, \vec{r}_{1} t_{0}\right)\left[-G\left(\vec{r}_{1} t, \vec{r}_{1} t_{0}\right) G\left(\vec{r}_{2} t, \vec{r}_{2} t_{0}\right)\right. \\
& \left.\left.+G\left(\vec{r}_{1} t, \vec{r}_{2} t_{0}\right) G\left(\vec{r}_{2} t, \vec{r}_{1} t_{0}\right)\right]\right\rangle \text { for } \quad \vec{r} \neq 0 .
\end{aligned}
$$

As a consequence of the static approximation, the site sum $\sum_{\vec{y}}$ has vanished from (4), and thus, is unfortunately no longer working to improve statistics.

The final correlator we use is extended from (6) to a $K \times K$ matrix by employing several levels $k=1 \ldots K$ of operator smearing. The procedure amounts to replacing in (11) all light-quark fields $\psi, \bar{\psi}$ with smeared fields $\psi^{\{k\}}, \bar{\psi}^{\{k\}}$. We have used Wuppertal-style fermion smearing [9] along with APE-style gauge field fuzzing [10]. The implementation specific to the asymmetric lattice used here is discussed below. No smearing, nor link variable fuzzing, was done for the heavy, static, quark fields in order to preserve spatial locality, i.e. the $\delta$ factor in (5). Thus, generically, replacing $\mathcal{O} \rightarrow \mathcal{O}^{\{k\}}=$ $\mathcal{O}\left[\psi^{\{k\}}, \bar{\psi}^{\{k\}} \ldots\right]$ the correlator (6) becomes a $K \times K$ matrix

$$
C^{k k^{\prime}}\left(t, t_{0}\right)\left(\vec{r} ; t, t_{0}\right)=\left\langle\mathcal{O}_{\mu}^{\{k\}}(\vec{r} ; t) \overline{\mathcal{O}}_{\mu}^{\left\{k^{\prime}\right\}}\left(\vec{r} ; t_{0}\right)\right\rangle,
$$

with $k, k^{\prime}=1 \ldots K$ and a sum over $\mu$ is understood. The expression for $C^{k k^{\prime}}\left(t, t_{0}\right)$ in terms of quark propagators still has the form given by (6), however, lightquark propagator elements are replaced with smeared ones, $G \rightarrow G^{\left\{k k^{\prime}\right\}}$. Smearing and fuzzing prescriptions are the same at source and sink. Thus the correlation matrix (7) is hermitian by construction.

We choose an asymmetric and anisotropic lattice with geometry $L_{1} \times L_{2} \times L_{3} \times L_{4}=8 \times 8 \times 32 \times 16$. The bare lattice constants, in the respective directions, satisfy $a_{1}=a_{2}=2 a_{3}=2 a_{4}$. Subsequently we shall refer to $a_{4}=a_{3}=a$ as the lattice constant $a$ of the simulation, so the physical volume is $16 a \times 16 a \times 32 a \times 16 a$. The lattice has a fine resolution in 4-direction (time), and the 
same fine resolution in the (spatial) 3-direction where the lattice size is twice as long as in the 1,2-directions. The idea here is to place the static quark sources along the 3 -direction and thus achieve a fine spatial resolution of the relative distance as well as to provide enough space to allow for asymptotic separation of the hadrons.

The spatial lattice asymmetry leads us to modify the procedure for operator smearing. A smearing iteration of $k$ steps is understood to mean $k$ steps in the 1,2-directions but $2 k$ steps in the 3 -direction, so the physical smearing extent is the same for all spatial directions (neglecting renormalization effects). Mutatis mutandis, the same is true for gauge field fuzzing. An illustration is shown in Fig. 3. The implementation used here is described in more technical terms in reference [11].

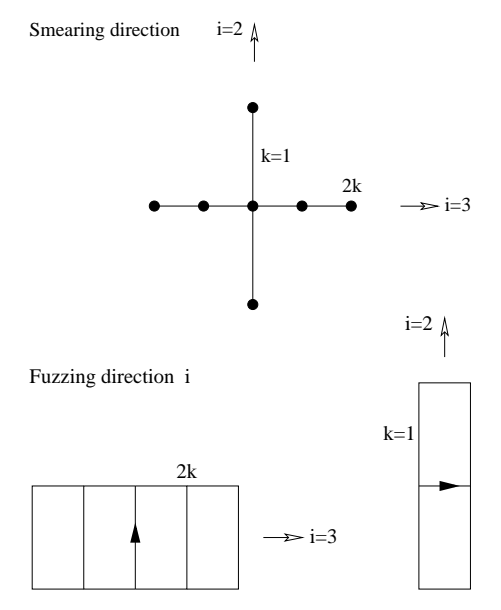

FIG. 3: Illustration of the prescription for quark field smearing (top) and gauge field fuzzing (bottom) on the asymmetric lattice. The geometry for $k=1$ iteration is shown.

The positions of the static quark sources are chosen as $x=(5,5, n, 3)$ with $n=1,2,3,4,8,11,13,17$. Their spatial locations along the 3 -direction, see Fig. 4 allow us to achieve any relative distance $r=1 a \ldots 16 a$ by choosing an appropriate pair of source points. Some relative distances can be obtained more than once. In those cases appropriate averages over sets of source and sink pairs have been taken to compute the correlator matrix elements. Because periodic boundary conditions are applied in the spatial directions relative distances larger than $r=16 a$ are redundant.

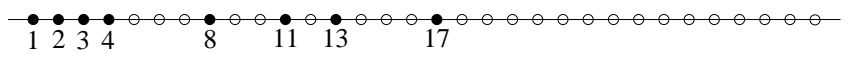

FIG. 4: Placement of static hadron sources along the 3direction of the lattice. Periodic boundary conditions are applied to the quark and gluon fields. All distances between $r=1 a$ and $r=16 a$ can be achieved, some multiple times.

Given the approximations employed in this work, e.g. static heavy quarks, and a few more mentioned below, it would be of no advantage to employ sophisticated im- proved lattice actions. We have therefore used the standard Wilson plaquette action with Wilson fermions in a quenched simulation [8]. The gauge field couplings in the $\mu-\nu$ planes and the hopping parameters in directions $\mu$ are given by, respectively,

$$
\beta_{\mu \nu}=\beta \frac{a_{1} a_{2} a_{3} a_{4}}{\left(a_{\mu} a_{\nu}\right)^{2}} \quad \text { and } \quad \kappa_{\mu}=\frac{\kappa}{a_{\mu} \frac{1}{4} \sum_{\nu=1}^{4} \frac{1}{a_{\nu}}} .
$$

The simulation was done at $\beta=6.2$ with four values $\kappa=0.140,0.136,0.132,0.128$ of the hopping parameter employing a multiple mass solver [12]. A total of 343 gauge field configurations were used.

\section{MASS SPECTRUM ANALYSIS}

The time evolution of the eigenvalues of $C\left(t, t_{0}\right)$ determines the energy spectrum. Eigenvalues, behaving exponentially with $t$, may rapidly vanish into numerical noise. Conventional diagonalization methods do not work well under those circumstances. Singular value decomposition (SVD), on the other hand, is ideally suited to the task 13]. The SVD reads

$$
C\left(t, t_{0}\right)=U\left(t, t_{0}\right) \Sigma\left(t, t_{0}\right) V^{\dagger}\left(t, t_{0}\right),
$$

where $U\left(t, t_{0}\right)$ and $V\left(t, t_{0}\right)$ are unitary in our case 27], and $\Sigma\left(t, t_{0}\right)=\operatorname{diag}\left(\sigma_{1}\left(t, t_{0}\right) \ldots \sigma_{K}\left(t, t_{0}\right)\right)$ contains the singular values satisfying $\sigma_{k}\left(t, t_{0}\right) \geq 0$. If $C\left(t, t_{0}\right)$ is non-degenerate and positive semi-definite then the set of singular values $\left\{\sigma_{k}\left(t, t_{0}\right): k=1 \ldots K\right\}$ and the set of eigenvalues are the same. A few more details can be found in [11]. For simplicity we will refer to $\sigma_{k}\left(t, t_{0}\right)$ as eigenvalues.

To extract energy levels from the eigenvalues an often used procedure, sometimes starting from a generalized eigenvalue problem [14], is to construct effective mass functions from the eigenvalues and then try to identify plateaus in the asymptotic time region. Typically, only a narrow subset of the time slices is usable, which also is subject to some discretion. We shall not rely on effective mass functions here, but rather analyze correlator eigenvalues by means of the maximum entropy method [15] following the adaption described in [11], and in [16]. Among the advantages are that all available time slices may be used, if desired, and that excited states, if present, are easily revealed.

The time dependence of the eigenvalues $\sigma_{k}\left(t, t_{0}\right)$ shall be fit with the model

$$
F(\rho \mid t)=\int_{0}^{\infty} d \omega \rho(\omega) e^{-\omega t} .
$$

for some set of discrete times slices $t=t_{1} \ldots t_{2}$, where $\rho(\omega)$ is a spectral density function. The maximum entropy method (MEM) is based upon Bayesian statistics. In this context the numbers $\rho(\omega)$ are interpreted 
as stochastic variables [15]. Their most likely values are obtained by simulated annealing as described in [1]. We refer the reader to this reference for technical details.

At each of the 16 relative distances $r$ we have used three $(K=3)$ operator smearing levels $k=1,2,3$. A typical set of eigenvalues is displayed in Fig. 5 for distance $r=1 a$ and the smallest (light) quark mass, $\kappa=0.140$. Only two eigenvalue correlators are shown, the third one is indistinguishable from zero due to numerical noise. Note that eigenvalues are separated by about four orders of magnitude. Thus crossing ambiguities are nonexistent [28]. The eigenvalue sets for all other distances $r$ exhibit the same features.

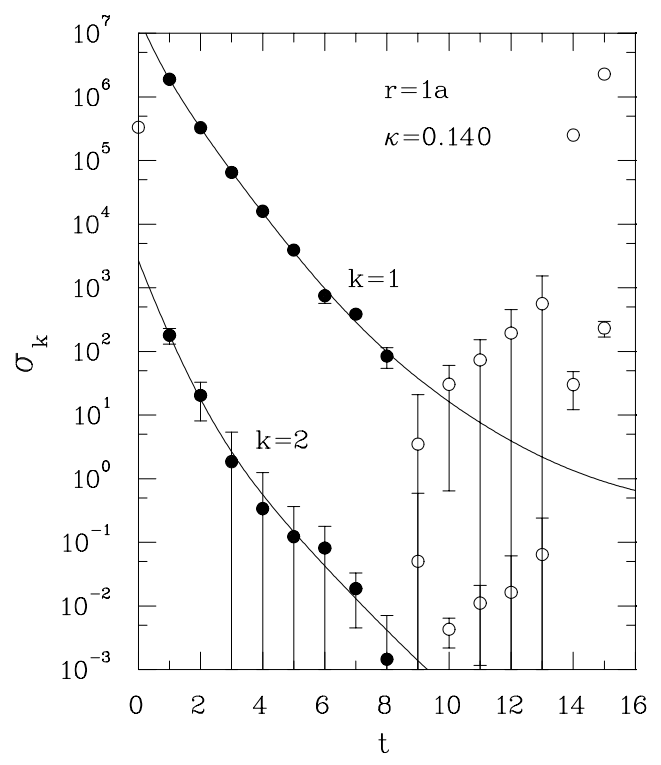

FIG. 5: Two sets of eigenvalues $\sigma_{k}\left(t, t_{0}\right), k=1,2$, of the $3 \times 3$ time correlation matrix (9) for relative distance $r=1 a$ and quark hopping parameter $\kappa=0.140$. The third eigenvalue, $k=3$, is not shown, it vanishes into numerical noise. Filled plot symbols belong to time slices used for the MEM fits employing the model (10), the curves are the corresponding results. Note that the source has been shifted to $t_{0}=0$.

The eigenvalue correlators were fit with the model (10) using, consistently in all cases, time slices $t=1 \ldots 8$ with the source $t_{0}=0$ being omitted, and mass range of $0 \leq \omega \leq 4$ with discretization interval size $\Delta \omega=0.05$, all in units of $a^{-1}$. The maximum entropy method then yields spectral density functions $\rho(\omega)$. Representative examples are shown in Fig. 6 for $r=1,4,8,16$, in units of $a$, and $\kappa=0.140$. The histogram lines in Fig. 6 represent $\rho(\omega)$, obtained from simulating annealing (cooling) following the very procedure put forward in [11]. Sixteen random annealing starts were used. The solid histogram lines represent the average of those 16 runs, the dashed histogram lines give the corresponding standard deviation. In most cases the spectra exhibit isolated peaks, say $\delta_{n}=\{\omega: \omega \in$ peak $\# n\}$. Then one may compute, for each peak $n$, the volume $Z$, the energy $E$, and the
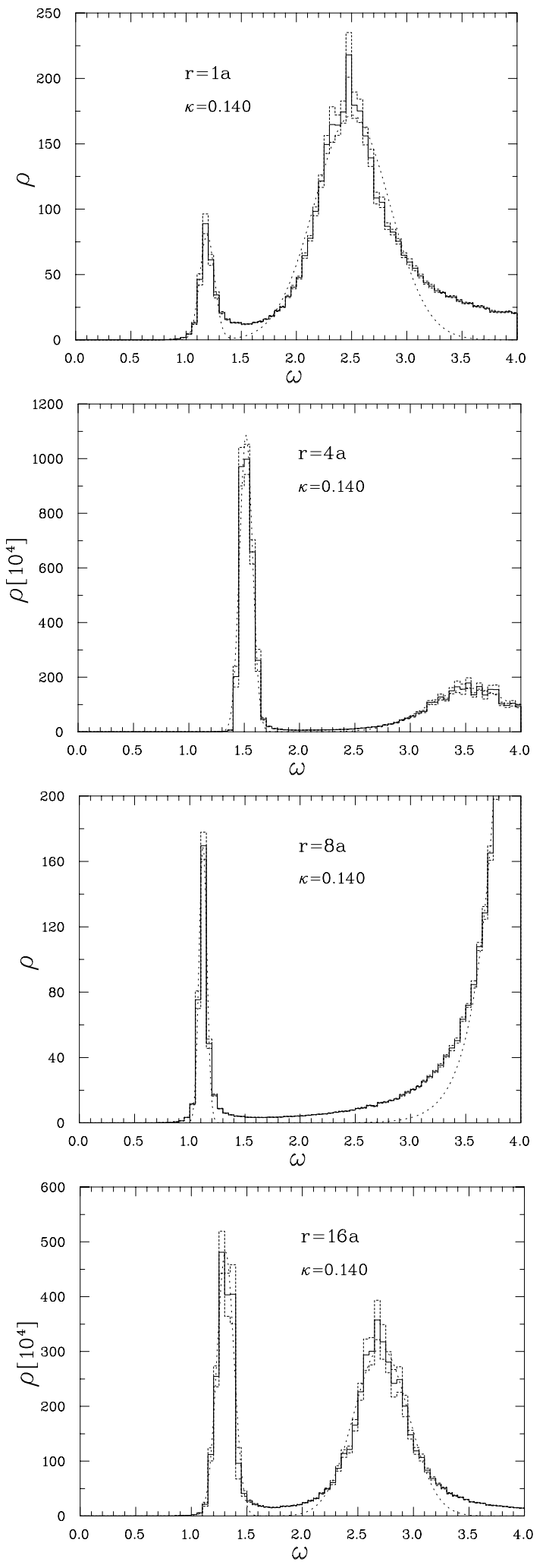

FIG. 6: Spectral density functions $\rho$, according to (10), for two-hadron operators with relative distance $r=1,4,8,16$, in units of $a$, thick histogram lines. Shown are eigenvalue spectra which contain ground state peaks. The dashed histogram lines indicate errors as explained in the text. The smooth dotted lines correspond to Gaussian fits to the discretized spectral densities. 
width $\Delta$, according to

$$
\begin{aligned}
Z & =\int_{\delta_{n}} d \omega \rho(\omega) \\
E & =Z^{-1} \int_{\delta_{n}} d \omega \rho(\omega) \omega \\
\Delta^{2} & =Z^{-1} \int_{\delta_{n}} d \omega \rho(\omega)(\omega-E)^{2} .
\end{aligned}
$$

Typically the largest eigenvalues reveal the ground state. There are few exceptions. The spectra at $r=1 a$ and $r=$ $8 a$, shown in Fig. 6, are examples. Selecting the support $\delta_{n}$ of a peak can be slightly ambiguous, particularly if the peak sits on background noise, or if there is overlap with another one. We have therefore, as a matter of course, performed least-square fits to the average spectral functions $\rho(\omega)$ with one or two Gaussians, as required in each case. These fits then give values for $Z, E$, and $\Delta$ as defined in (11)-(13) directly from the parameters of the Gaussians. In this manner we have obtained 16 sets, one for each $r$, of five-quark hadron ground state energies. Each set contains four energies corresponding to hopping parameter values $\kappa=0.140,0.136,0.132,0.128$.

The spectral density functions $\rho(\omega)$ typically exhibit some additional structure at the high end of the $\omega$ range, a broad peak in most cases. These secondary peaks appear because diagonalizing the correlator matrix separately on all time slices only ensures that its eigenvalues describe a single state from the physical spectrum at large values for $t$ [14]. At small time slices, close to the source, the eigenvectors of $C\left(t, t_{0}\right)$ may still describe a mix of spectral states. In practice the large- $t$ rule means that, in each eigen channel, one should only take the lowest peak into account. In physical terms the secondary peaks are separated from those by $\approx 3 \mathrm{GeV}$, and larger, and thus should probably be considered lattice artifacts.

In order to relate our results to the physical hadron spectrum we have also computed $3 \times 3$ correlation matrices with standard local [8] pseudoscalar meson $(\pi)$, vector meson $(\rho)$, and nucleon $(N)$ operators, using the same smearing and fuzzing prescription. The analysis was performed with the MEM in exactly the same way as described above. This now allows extrapolations of hadron masses to the physical pion mass region $\left(a m_{\pi} \approx 0\right)$. We use the extrapolation model introduced in [1]

$$
y=c_{1}+c_{2} x+c_{3} \ln (1+x), \quad x=\left(a m_{\pi}\right)^{2},
$$

and $y=a m$. For a motivation of the same we refer the reader to said reference. The extrapolation prescription is, of course, a source of systematic error on our results. In Fig. 7 we show the vector meson and nucleon masses as a function of the squared pion mass and the extrapolations according to (14). The extrapolated values $a m_{\rho}=0.293$ and $a m_{N}=0.531$, as $x \rightarrow 0$, are used to set the reduced mass of the $\rho N$ system to its experimental value $m_{\rho N}=424.7 \mathrm{MeV}$. This results in a lattice
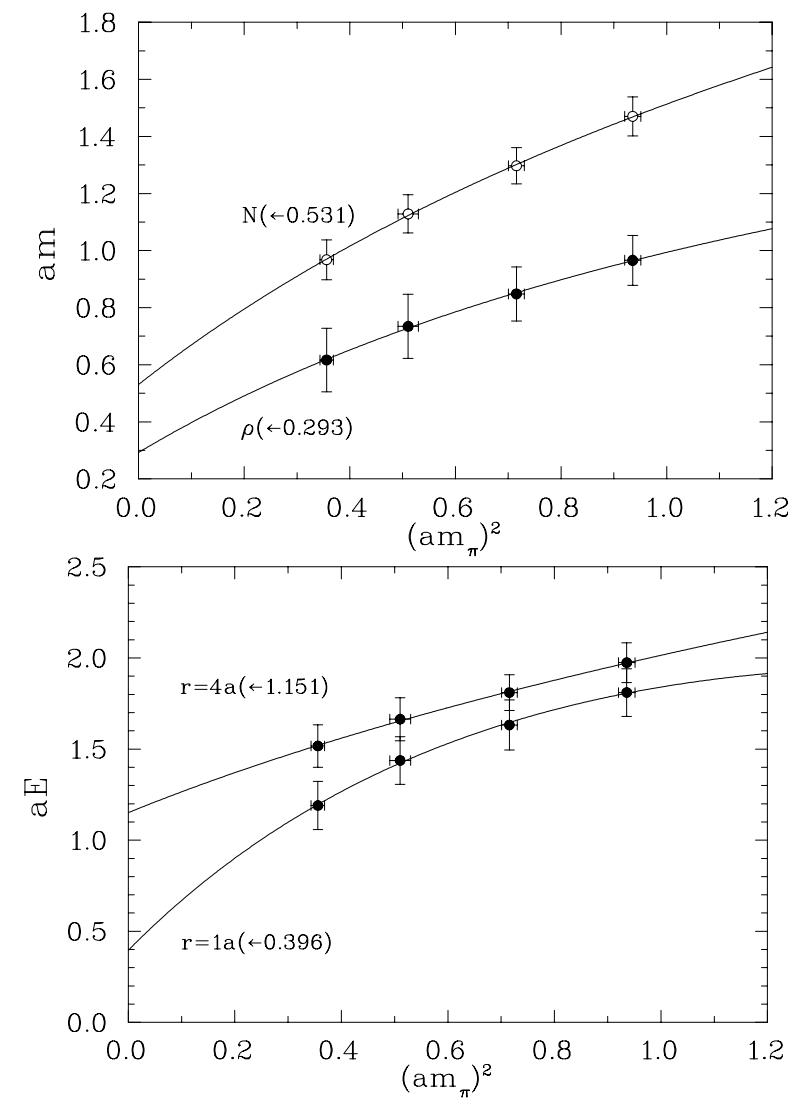

FIG. 7: Extrapolations of the vector meson and nucleon masses $y=a m$ based on the model (14) and four hopping parameter values, top panel. The bottom panel shows two typical examples of five-quark hadron ground state energies $y=a E$, at distances $r=1 a$ and $r=4 a$, and their respective extrapolation using the same procedure.

constant of $a^{-1}=2251 \mathrm{MeV}$ or $a=0.088 \mathrm{fm}$. The corresponding vector meson and nucleon masses come out as $659 \mathrm{MeV}$ and $1196 \mathrm{MeV}$, respectively, which deviate by $15 \%$ and $27 \%$ from their experimental values. We take these numbers as indicators of the systematic errors to be expected due to the extrapolation.

Also in Fig. 7 two representative examples of extrapolations of five-quark hadron ground state masses $y=a E$ are shown, the relative distances are $r=1 a$ and $r=4 a$. The error bars represent spectral peak widths $\Delta$ according to (13). We utilize those to compute uncertainties on the extrapolated energies: At each of the four data points stochastically independent Gaussian random numbers with average and standard deviation given by the energies and peak widths of the data, respectively, were generated and again fitted with (14). Repeating this a large number of times then gives rise to a standard deviation which we interpret as a MEM peak extrapolated width $\Delta$. The extrapolated energy spectrum obtained in this way is displayed in Fig. 8 as a function of the relative distance $r$. 


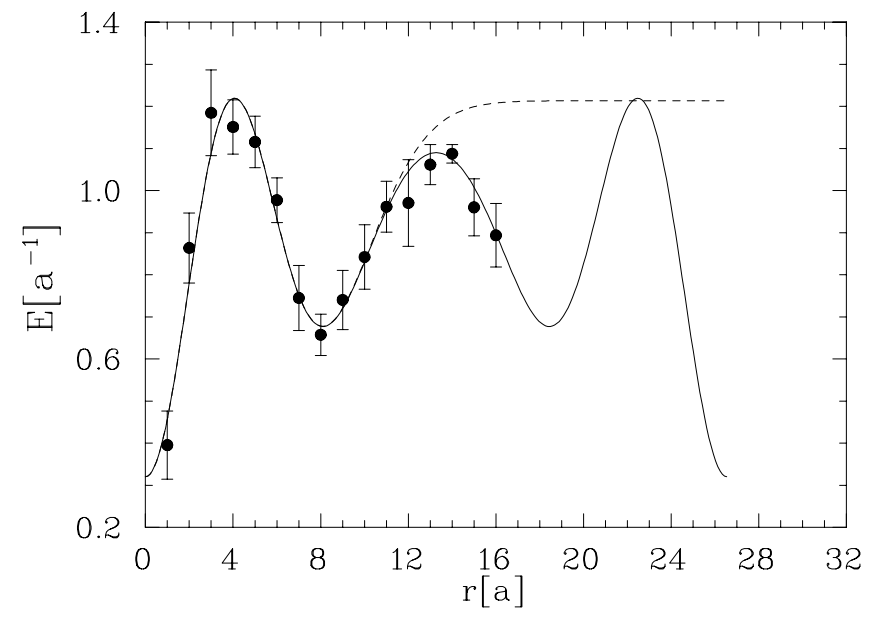

FIG. 8: Extrapolated ground-state energy $E$ of the heavylight $K-\Lambda$ like system versus the relative distance $r$ with MEM based uncertainties (plot symbols). The solid line represents a fit with the model (16). The dashed line shows the same fit, but the mirror term $a V(L-x)$ has been dropped.

The energies shown in Fig. 8 are also subject to statistical errors which originate from the gauge configuration ensemble. To identify those a bootstrap error analysis 17] was performed. Using a resampling factor of eight on the 343 gauge configurations the MEM spectral analysis was repeated for each bootstrap sample 29]. In each case the same fixed random number sequence was employed in order to eliminate fluctuations in the annealing process. The statistical errors $S$ thus obtained are compared to the peak widths $\Delta$ in Tab. I] Results are for $\kappa=0.140$ (lightest quark mass). The other $\kappa$ values give very similar numbers because the underlying set of gauge configurations is the same. Clearly the statistical errors $S$ are consistently smaller than the peak widths $\Delta$. On average over all relative distances $r$ the ratio $\Delta / S$ turns out to be slightly larger than two. Thus, judiciously keeping the larger errors, we have used the spectral peak widths as the principle input for computing uncertainties.

\section{ADIABATIC POTENTIAL}

The data points in Fig. 8 connect smoothly along a continuous curve. In order to find an appropriate fit model we note that the ground-state $K-\Lambda$ like system should be in a relative S-wave. Our choice thus are Swave $3 \mathrm{~d}$ harmonic oscillator functions [18]. The first few terms are given by

$$
a V(x)=\exp \left(-\alpha_{1} x^{2}\right)\left(\alpha_{2}+\alpha_{3} x^{2}+\alpha_{4} x^{4}\right), \quad x=r / a,
$$

with parameters $\alpha_{1} \ldots \alpha_{4}$. This will not be sufficient though for two reasons. First, periodic boundary conditions have been imposed on the lattice in the 3-direction.
TABLE I: Comparison of the statistical errors $S$ of the ground-state peak positions $E$, see (12), and the peak widths $\Delta$, see (13). Results are shown for the lightest quark mass, $\kappa=0.140$, and for all relative distances $r$.

\begin{tabular}{rccc}
\hline \hline$r / a$ & $a E$ & $a \Delta$ & $a S$ \\
\hline 1 & 1.193 & 0.050 & 0.038 \\
2 & 1.434 & 0.106 & 0.031 \\
3 & 1.514 & 0.083 & 0.037 \\
4 & 1.526 & 0.062 & 0.059 \\
5 & 1.512 & 0.067 & 0.076 \\
6 & 1.378 & 0.053 & 0.032 \\
7 & 1.206 & 0.052 & 0.024 \\
8 & 1.120 & 0.035 & 0.029 \\
9 & 1.190 & 0.055 & 0.030 \\
10 & 1.223 & 0.065 & 0.020 \\
11 & 1.348 & 0.066 & 0.034 \\
12 & 1.390 & 0.072 & 0.030 \\
13 & 1.465 & 0.047 & 0.024 \\
14 & 1.338 & 0.080 & 0.030 \\
15 & 1.315 & 0.058 & 0.040 \\
16 & 1.297 & 0.062 & 0.009 \\
\hline \hline
\end{tabular}

Thus lattice sites with relative distances $x$ and $L_{3}-x$ are equivalent. However, as the two-hadron operators for the $K-\Lambda$ like system are essentially loop operators, see Fig. 2], those do not share the periodic behavior of the fundamental lattice fields. This effect should be most notable for distances around $\approx L_{3} / 2=16$. Second, interactions with hadron images in adjacent copies of the lattice are present. Their dominant effect is a constant interaction energy, as the relative distances of most of the mirror images remain constant with varying $x$. Thus motivated, empirically one finds that the lattice data are well represented by the model

$$
a V_{L}(x)=\alpha_{0}+a V(x)+a V(L-x), \quad x=r / a,
$$

where $\alpha_{0}$ and $L$ are additional parameters. The resulting fit is shown in Fig. 8 as a solid line. The dashed line represents $\alpha_{0}+a V(x)$ only. Table $\Pi$ contains the fit parameters. Note that $L$ deviates somewhat from 32 , as should

TABLE II: Results for the fit parameters using the model (16).

\begin{tabular}{cc}
\hline \hline$\alpha_{0}$ & $1.213(93)$ \\
$\alpha_{1}$ & $0.0409(37)$ \\
$\alpha_{2}$ & $-0.89(10)$ \\
$\alpha_{3}$ & $0.109(19)$ \\
$\alpha_{4}$ & $-0.00328(52)$ \\
$L$ & $26.54(57)$ \\
\hline \hline
\end{tabular}

be expected for the reason stated above. The $\chi^{2}$ per degree of freedom is 0.63 , the uncertainties given stem from the covariance matrix of the Levenberg-Marquardt algorithm. We list those for completeness only, they will not be used in the subsequent analysis. Rather, because we 
are mostly interested in uncertainties of possible bound state energies, as the case may be, we proceed as follows: Each data point in Fig. 8 is randomized by stochastically independent Gaussian random numbers with average and standard deviation given by the energies and peak widths of the data, respectively. Then the fit with the model (16) is repeated, the corresponding set of parameters gives rise to a new potential $V(x)$. The procedure is performed 32 times. We show the results in Fig. 9] along with the potential obtained directly from the original data points. Specifically, Fig. 9 displays adiabatic potentials

$$
V_{a}(r)=V(r / a)
$$

on physical scales given by the lattice constant $a$, as determined above.
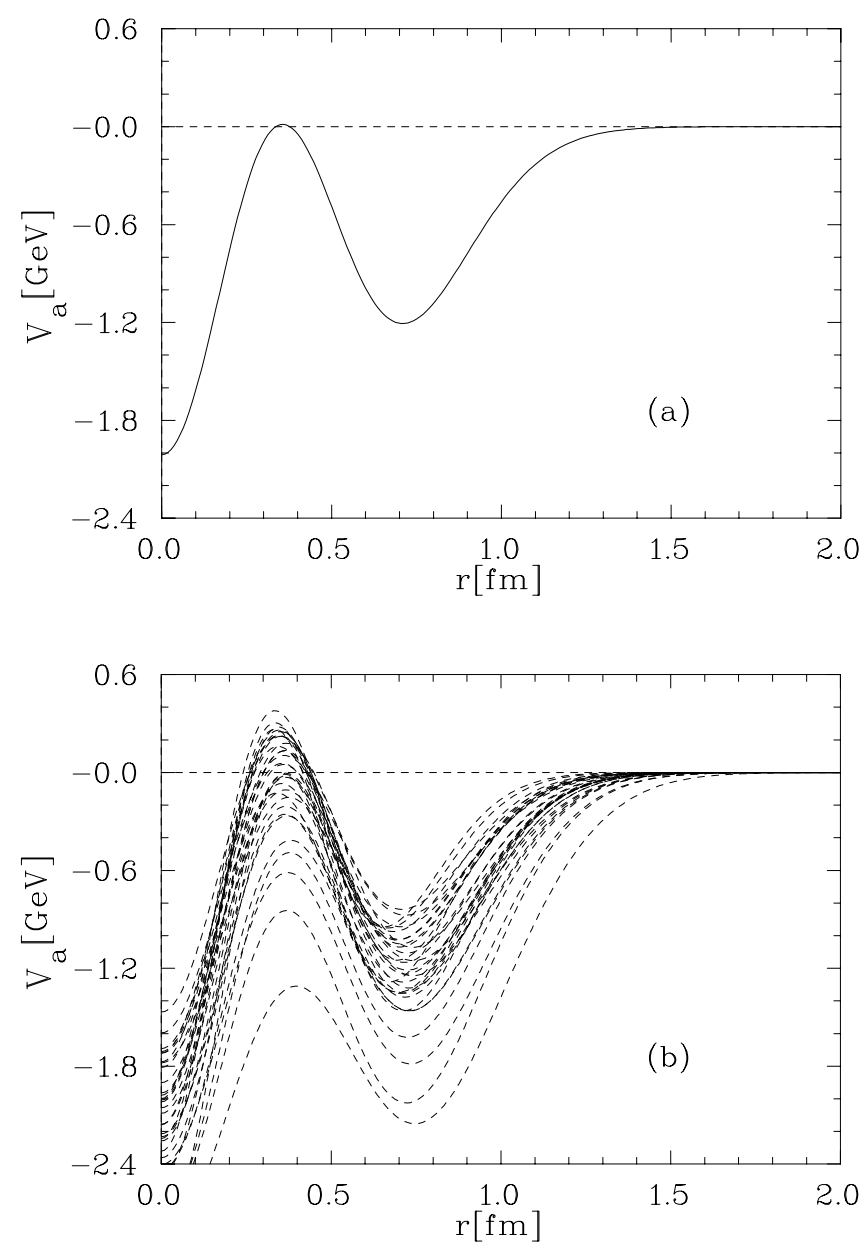

FIG. 9: Adiabatic potential $V_{a}(r)$ resulting from the lattice simulation in physical units, panel (a). Panel (b) shows potentials from 32 sets of randomizations of the ground state energies $E$ as seen in Fig. 8 .

\section{BOUND STATES AND WAVE FUNCTIONS}

Figure 9 represents the end result of the lattice simulation. Although we expect that systematic errors coming from extrapolations may be as large as 30\%, and the uncertainties on the potential itself according to Fig. 9(b) are sizable, we believe that important physics can still be learned on a qualitative level. First, we observe that $V_{a}(r)$ of Fig. 9(a) has no repulsive regions. This is a reasonable, and confidence inspiring, outcome because repulsion in a two-hadron system is typically Pauli repulsion, but of course the latter is absent in a $K-\Lambda$ like system.

Also, as mentioned earlier just before Eqn. (6), the relative distance $r=0$ was excluded from the simulation because "color twisted" operators would then have to be included for all distances $r$, to be consistent. Without further work it remains an open question how such a family of operators might influence the form of $V_{a}(r)$. However, it seems reasonable to assume that those will not play a major role for large $r$ because there they would create hadrons inconsistent with confinement, whereas at $r=0$ mixing with those states would lower the ground state energy according to general principles of quantum mechanics. Thus, we believe that the current simulation correctly captures the most important physics of the fourquark system.

The salient features of $V_{a}(r)$ are the two distinct regions of attraction around $r \sim 0.5-1.0 \mathrm{fm}$ and $r \lesssim 0.2 \mathrm{fm}$. An obvious question is whether the attraction is sufficient to generate bound states of the five-quark molecule. To find out we have, first, computed scattering phase shifts $\delta_{\ell}(p)$ employing the adiabatic potential $V_{a}(r)$ in a Schrödinger equation. For the reduced mass $m$ we chose, in turn, the three values of the physical $K-\Lambda, D-\Lambda_{c}$, and $B-\Lambda_{b}$ systems [3]. These evaluate to $a m=0.152,0.415$, and 1.172 , respectively. Continuum boundary conditions were implemented by solving a Volterra integral equation within the Jost function formalism [19]. The resulting scattering phase shifts are shown in Fig. 10. According to Levinson's theorem, see [19] for example, we have $\delta_{\ell}(0)-\delta_{\ell}(\infty)=n \pi$ where $n$ is the number of bound states. In the S-wave $n$ is one for the $K-\Lambda$ system, two for the $D-\Lambda_{c}$ system, and four for the $B-\Lambda_{b}$ system. We need to caution though that relativistic effect are large in $K-\Lambda$, with increasing quark mass $(s \rightarrow c \rightarrow b)$ this becomes less of a concern. The scattering phase shifts are quite featureless, but they give us an exact count of the number of bound states present in the simulation.

In order to compute bound state energies and wave functions we solve the radial Schrödinger equation with $V_{a}(r)$. Our conventions can be gleaned from

$$
-\frac{d^{2} u_{a}}{d r^{2}}+\left[2 m V_{a}(r)+\frac{\ell(\ell+1)}{r^{2}}\right] u_{a}=2 m E u_{a}
$$




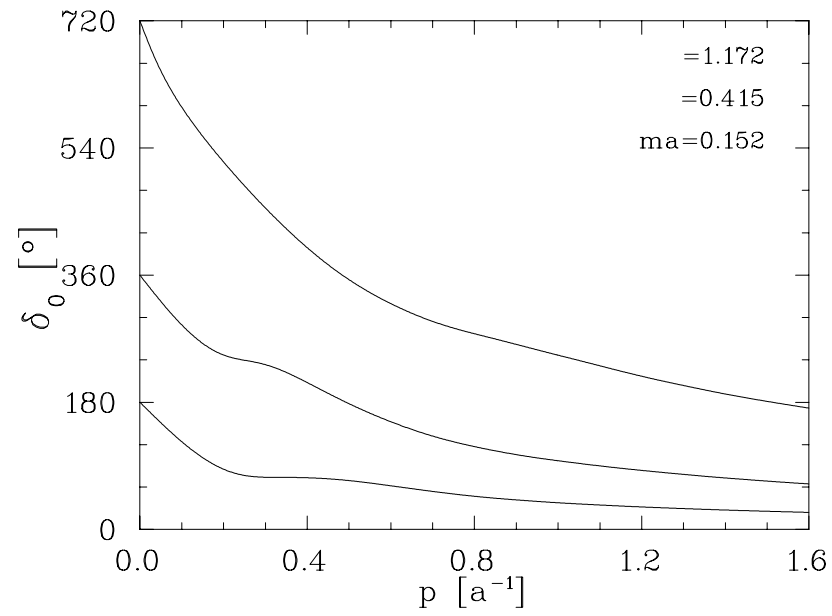

FIG. 10: Scattering phase shifts (S-wave) for the $K-\Lambda, D-$ $\Lambda_{c}$, and $B-\Lambda_{b}$ systems (bottom to top, respectively) versus the relative momentum $p$.

and

$$
\int_{0}^{\infty} d r\left|u_{a}(r)\right|^{2}=1 .
$$

The region between $r=0$ and $r=30 a$ was subdivided into 60 discretization intervals. A suitable cubic B-spline representation [20] for $u_{a}(r)$ was employed to enforce bound-state boundary conditions and provide smooth interpolation between the knots. This then leads to a generalized matrix eigenvalue problem. The scale neutral bound state energies are compiled in Tab. III for the three reduced masses of $K-\Lambda, D-\Lambda_{c}, B-\Lambda_{b}$, and three partial waves. The errors come from repeating the calculation with 32 randomizations of the potential, as seen in Fig. 9(b).

TABLE III: Scale neutral bound state energies $a E_{n}$ of the adiabatic potential $V_{a}(r)$ for three values $a m$ of the reduced mass $m$ and partial waves $\ell$. The reduced masses correspond to $K-\Lambda, D-\Lambda_{c}$, and $B-\Lambda_{b}$.

\begin{tabular}{cccccc}
\hline \hline$\ell$ & $a m$ & $a E_{1}$ & $a E_{2}$ & $a E_{3}$ & $a E_{4}$ \\
\hline 0 & 0.152 & $-0.22(12)$ & & & \\
& 0.415 & $-0.32(13)$ & $-0.04(13)$ & & \\
& 1.172 & $-0.39(13)$ & $-0.26(16)$ & $-0.15(12)$ & $-0.001(88)$ \\
\hline 1 & 0.152 & $-0.11(11)$ & & & \\
& 0.415 & $-0.28(13)$ & & & \\
& 1.172 & $-0.38(13)$ & $-0.14(12)$ & & \\
\hline 2 & 0.152 & & & & \\
& 0.415 & $-0.20(13)$ & & \\
& 1.172 & $-0.35(13)$ & $-0.11(12)$ & \\
\hline \hline
\end{tabular}

The S-wave bound state wave functions $u_{a}(r)$ and their squares $\left|u_{a}(r)\right|^{2}$ are displayed in Figs. 11112 and 13, The wave function of the $K-\Lambda$ system, Fig. 11, peaks at around $r \sim 0.6-0.9 \mathrm{fm}$. This is a typical nuclear physics distance scale, and clearly points to the structure of a

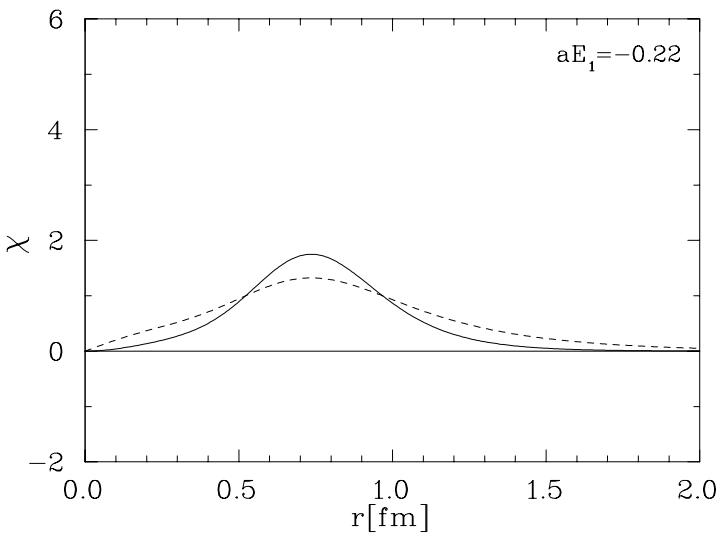

FIG. 11: S-wave bound state wave function $\chi=u_{a}(r)$ (dashed line) and its square $\chi=\left|u_{a}(r)\right|^{2}$ (solid line) for the $K-\Lambda$ system.

hadronic molecule. With increasing (heavy) quark mass the number of bound states also increases. The ground state of the $D-\Lambda_{c}$ system, Fig. 12, exhibits the same feature. However, the excited state wave function has sizable support at small distances $r \lesssim 0.2 \mathrm{fm}$. The wave function starts to 'feel' the short-range attraction of the potential $V_{a}(r)$, see Fig. 9(a). This property is fully developed in $B-\Lambda_{b}$, see Fig. 13. The ground state wave function 'lives' in the large-distance trough of $V_{a}(r)$ and thus points to a hadronic molecule. On the other hand, the wave function of first excited state 'lives' in the smalldistance regime of $V_{a}(r)$, which indicates a very tight spacial structure. There is nothing as distinct about the third state, and the forth one strongly resembles a continuum state.

The wave functions of the ground and first excited $B$ $\Lambda_{b}$ states with $a E_{1}=-0.39$ and $a E_{2}=-0.26$, respectively, shown in Fig. 13 deserve further comment. Viewing the system as consisting of five quarks, the ground state is clearly a pure two-hadron molecule, and the rmsradius evaluates to $r_{\mathrm{rms}} \approx 0.74 \mathrm{fm}$. The excited state, on the other hand, is spatially compact in a dramatic way: Most of the probability density $\left|u_{a}(r)\right|^{2}$ is within a region of less than $0.3 \mathrm{fm}$ radius and peaks just above $0.1 \mathrm{fm}$. Inevitably, this five-quark state should be interpreted as a pentaquark.

We should remark here that our original five-quark operators (11) are indeed capable of describing such physics, because the set comprises operators where the heavy quark and anti-quark have very small distances, the smallest one being the lattice constant $a=0.088 \mathrm{fm}$. Also, the $B-\Lambda_{b}$ results are probably our most reliable ones, because relativistic effects are not very important.

On the other hand, systematic errors, having to do with the adiabatic approximation, extrapolations to zero pion mass, and dismissal of relativistic effects, clearly render our interpretation a qualitative one. It is interesting to ask though if the reason for our inability to establish 

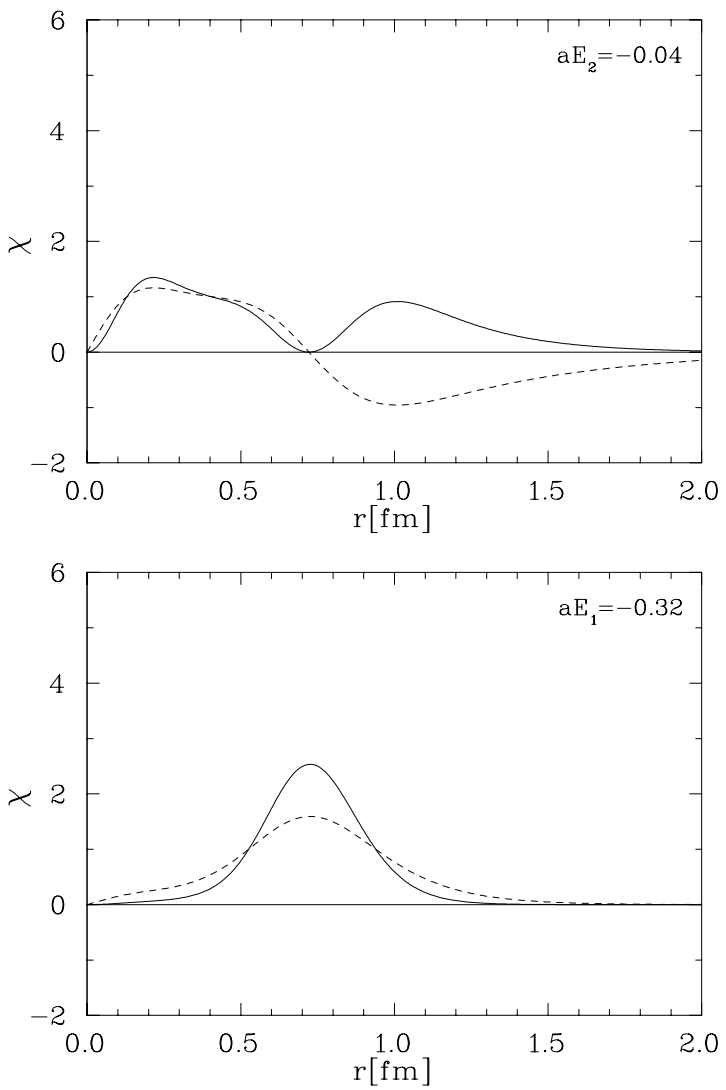

FIG. 12: S-wave bound state wave functions $\chi=u_{a}(r)$ (dashed lines) and their squares $\chi=\left|u_{a}(r)\right|^{2}$ (solid lines) for the $D-\Lambda_{c}$ system.

a clear experimental signal for a pentaquark 21, 22], and also to irrefutably establish a lattice QCD pentaquark 23, 24, 25], could be rooted in the assumption that the pentaquark shall be a ground state of bound light quarks. There is no a priori reason for this. The well-publicized quark model decouplet $\Theta$ particle [4] may well have a molecule-like structure.

Thus we speculate that the pentaquark, if we understand it as a tight five-quark bound state, may not exist for all light flavored $(\mathrm{u}, \mathrm{d}, \mathrm{s})$ quarks, but there is still hope in the heavy-flavor sector. However, a pentaquark may then reveal itself only as an excited state, the ground state being a hadronic molecule.

Finally, as a matter of course, we show in Figs. 14 and 15 the $P$ - and $D$-state wave functions of the calculation. And in Tab. IV we have compiled physical mass spectra of the considered five-quark systems by subtracting the computed binding energies from the experimental single meson and baryon masses 3]. We give those numbers for completeness only, without claiming quantitative relevance.
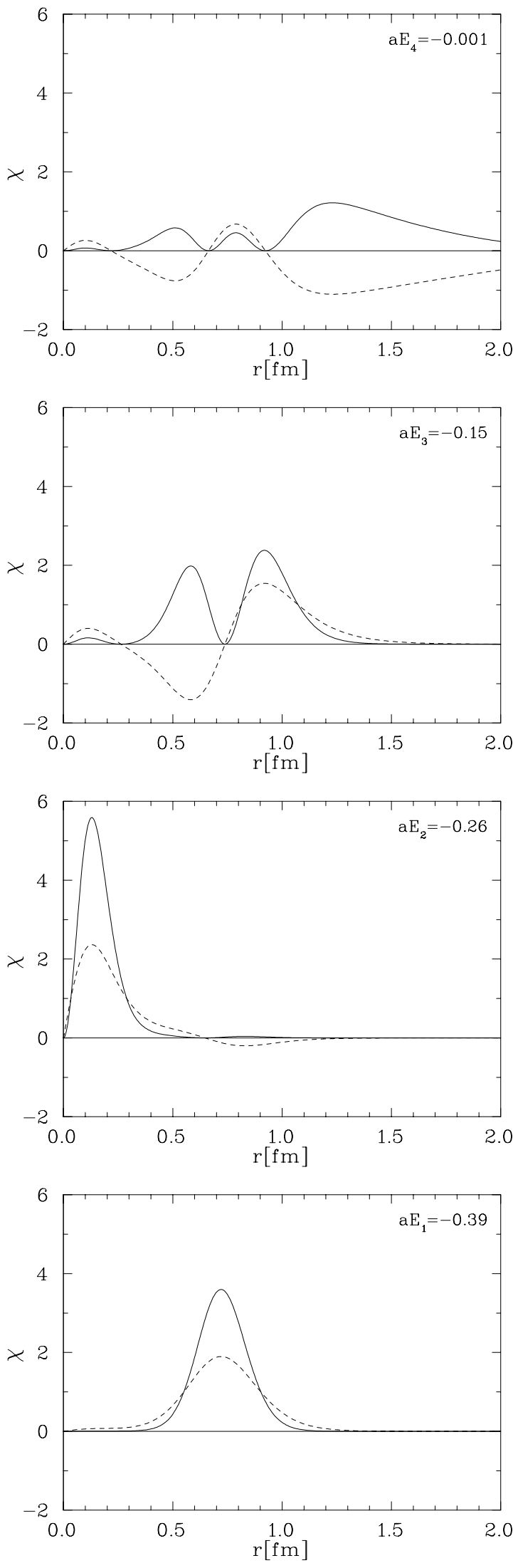

FIG. 13: S-wave bound state wave functions $\chi=u_{a}(r)$ (dashed lines) and their squares $\chi=\left|u_{a}(r)\right|^{2}$ (solid lines) for the $B-\Lambda_{b}$ system. 

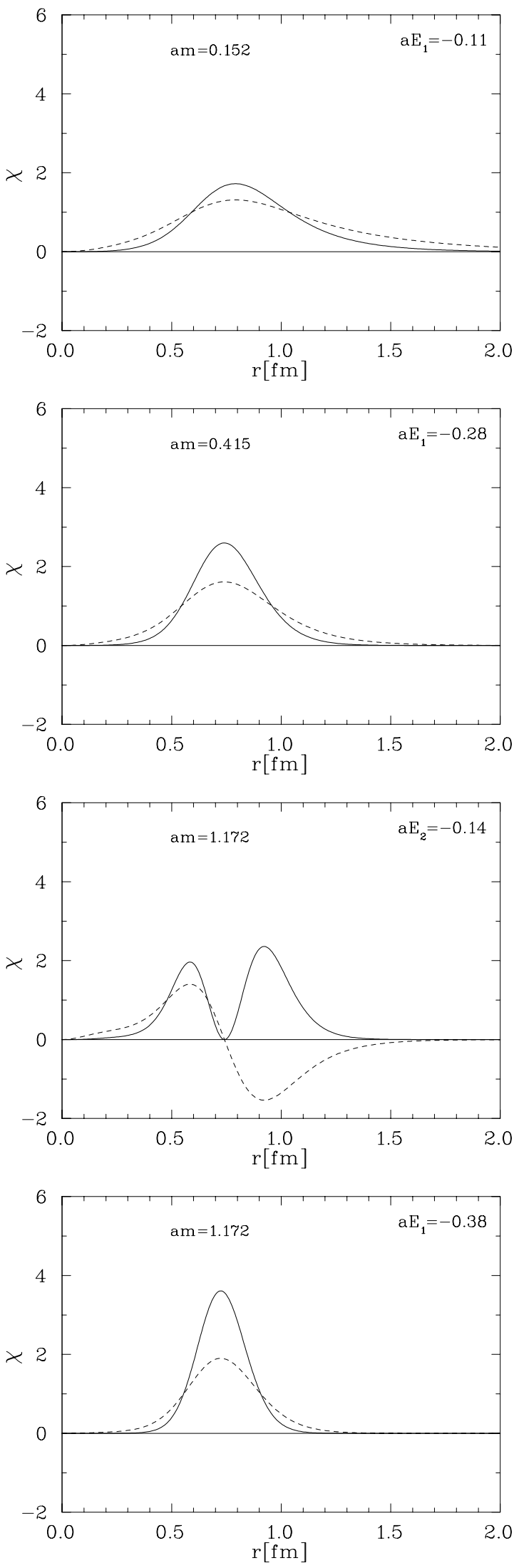

FIG. 14: P-wave bound state wave functions $\chi=u_{a}(r)$ (dashed lines) and their squares $\chi=\left|u_{a}(r)\right|^{2}$ (solid lines). The insets for $a m$ and $a E_{n}$ match the entries of Tab. III
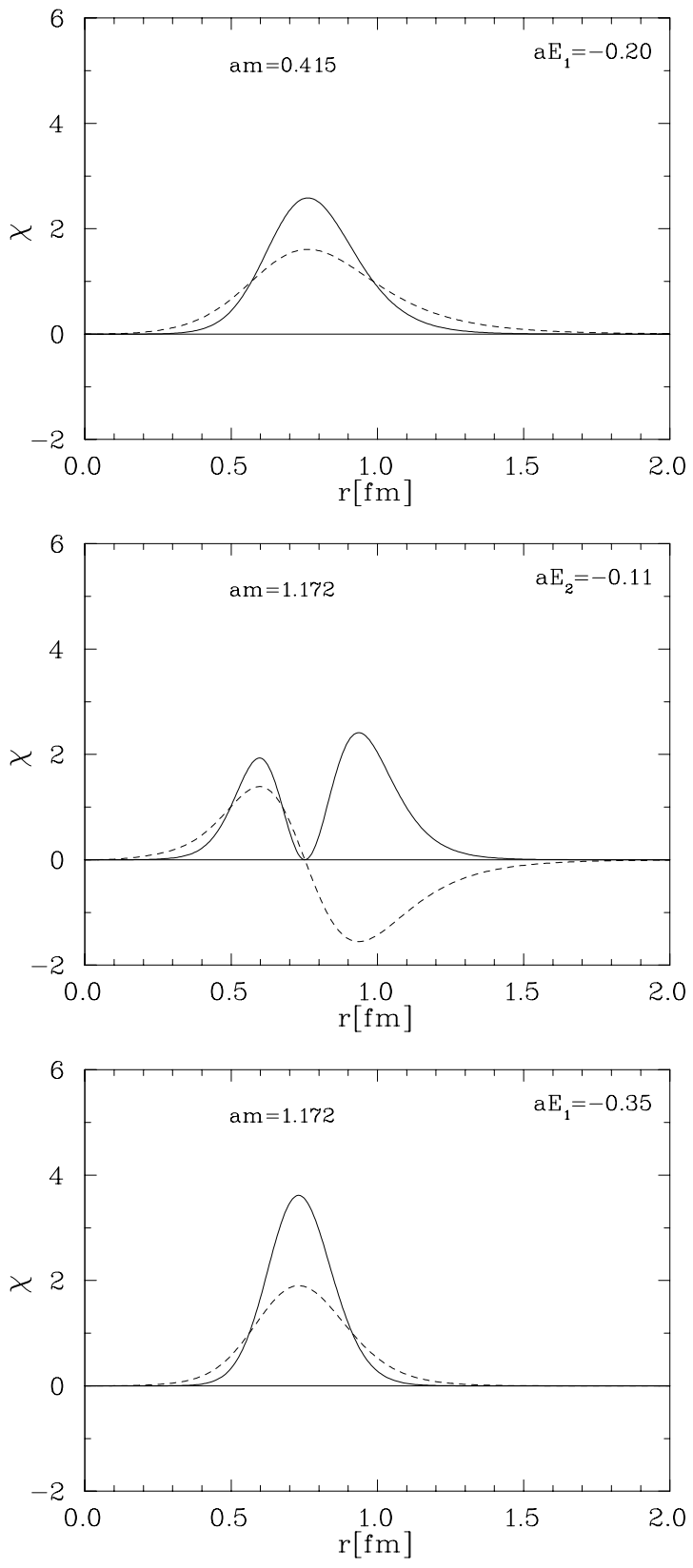

FIG. 15: D-wave bound state wave functions $\chi=u_{a}(r)$ (dashed lines) and their squares $\chi=\left|u_{a}(r)\right|^{2}$ (solid lines). The insets for $a m$ and $a E_{n}$ match the entries of Tab. III.

\section{SUMMARY AND CONCLUSION}

We have performed a lattice simulation of a five-quark $K-\Lambda$ like hadronic system on an anisotropic and asymmetric lattice. The heavy quark anti-quark pair was treated as static. Thus it was possible to compute the total energy of the system as a function of the relative distance $r$ between the hadrons. The maximum entropy method was employed toward this end. An extrapolation into the physical pion mass region was also performed. The objective was to extract an adiabatic potential $V_{a}(r)$ 
TABLE IV: Physical five-quark hadron masses $m_{n}$, in $\mathrm{GeV}$, for three values of the reduced mass $m$ corresponding to the systems $K-\Lambda, D-\Lambda_{c}$, and $B-\Lambda_{b}$. The layout matches that of Tab. III]

\begin{tabular}{cccccc}
\hline \hline$\ell$ & $m$ & $m_{1}$ & $m_{2}$ & $m_{3}$ & $m_{4}$ \\
\hline 0 & 0.342 & $1.11(28)$ & & & \\
& 0.934 & $3.44(29)$ & $4.07(29)$ & & \\
& 2.639 & $10.02(30)$ & $10.33(35)$ & $10.58(27)$ & $10.90(20)$ \\
\hline 1 & 0.342 & $1.36(26)$ & & & \\
& 0.934 & $3.54(29)$ & & & \\
& 2.639 & $10.05(30)$ & $10.60(27)$ & & \\
\hline 2 & 0.342 & & & & \\
& 0.934 & $3.70(29)$ & & \\
& 2.639 & $11.11(30)$ & $10.66(26)$ & & \\
\hline \hline
\end{tabular}

for the relative motion. The potential turns out to have two distinct attractive troughs, one at intermediate distances, $r \approx 0.7 \mathrm{fm}$, and one at short range, $r \lesssim 0.2 \mathrm{fm}$.

To study the dynamics of the two-hadron system we have used $V_{a}(r)$ in a Schrödinger equation for three values of the physical reduced mass, corresponding to $K-$ $\Lambda, D-\Lambda_{c}$, and $B-\Lambda_{b}$. With increasing heavy-quark mass $m_{s}<m_{c}<m_{b}$ the number of S-wave bound states increases from one to four. We have examined the corresponding wave functions to study the nature of the fivequark systems.

Systematic errors predominantly originating from using the adiabatic approximation, extrapolation to zero pion mass, the non-relativistic framework, and possibly the quenched approximation, render the results of our study qualitative only. Nevertheless, we believe that the results for the $B-\Lambda_{b}$ ground and excited state wave functions, respectively, bring to light a particularly interesting scenario with regard to five-quark system physics: The ground state is best described as a hadronic molecule, with a relative hadron-hadron distance matching a nuclear physics scale, whereas the excited state exhibits a wave function with support on very short distances of $r \lesssim 0.2 \mathrm{fm}$, or so. This leads us to interpret the excited state as a pentaquark. The results for the $b$ quark system are less prone to relativistic corrections and therefore are our most reliable ones. The light-quark $K_{-}$ $\Lambda$ exhibits one ground state which clearly is a hadronic molecule.

In the light of our results it might be worthwhile to initiate future lattice QCD studies with this scenario in mind, but with a more realistic set of operators, lattices, actions, and lighter quark masses, etc. All things considered, there is ground for the hypothesis that an all light-quark pentaquark may not exist, but that the existence of a genuine pentaquark in the heavy-quark sector cannot be ruled out. However, if present, it may well be an excited state.

This material is based upon work supported by the National Science Foundation under Grant No. 0300065.
[1] H. R. Fiebig and H. Markum, in Hadronic Physics from Lattice QCD, edited by A. M. Green (World Scientific, Singapore, 2004), vol. 9 of International Review of $\mathrm{Nu}$ clear Physics, chap. 4.

[2] M. Savage, in 23rd International Symposium on Lattice Field Theory, 25-30 July 2005, Trinity College, Dublin, Ireland, PoS(LAT2005)020 (2005), hep-lat/0508048.

[3] W. M. Yao et al. (Particle Data Group), J. Phys. G33, 1 (2006).

[4] D. Diakonov and V. Petrov, Phys. Rev. D69, 094011 (2004), hep-ph/0310212.

[5] D. Arndt, S. R. Beane, and M. J. Savage, Nucl. Phys. A726, 339 (2003), nucl-th/0304004.

[6] C. Michael and P. Pennanen (UKQCD), Phys. Rev. D60, 054012 (1999), hep-lat/9901007.

[7] A. Mihály, H. R. Fiebig, H. Markum, and K. Rabitsch, Phys. Rev. D55, 3077 (1997).

[8] I. Montvay and G. Münster, Quantum Fields on the Lattice (Cambridge University Press, Cambridge, UK, 1994).

[9] C. Alexandrou, S. Güsken, F. Jegerlehner, K. Schilling, and R. Sommer, Nucl. Phys. B414, 815 (1994), heplat/9211042.

[10] C. Albanese et al., Phys. Lett. B192, 163 (1987).

[11] M. S. Cook and H. R. Fiebig, Phys. Rev. D74, 094501 (2006), hep-lat/0609010.

[12] U. Glässner, S. Güsken, T. Lippert, G. Ritzenhöfer, K. Schilling, and A. Frommer, Int. J. Mod. Phys. C7, 635 (1996), hep-lat/9605008.

[13] G. H. Golub and C. F. Van Loan, Matrix Computations (Johns Hopkins University Press, Baltimore, MD, 1996), 3rd ed., iSBN 0-8018-5413-X.

[14] M. Lüscher and U. Wolff, Nucl. Phys. B339, 222 (1990).

[15] M. Jarrell and J. E. Gubernatis, Phys. Rep. 269, 133 (1996).

[16] H. R. Fiebig, Phys. Rev. D65, 094512 (2002), heplat/0204004.

[17] B. Efron, Annals of Statistics 7, 1 (1979).

[18] J. D. Talman, Nucl. Phys. A 141, 273 (1970).

[19] J. R. Taylor, Scattering Theory (Wiley, New York, 1972).

[20] C. de Boor, A Practical Guide to Splines (SpringerVerlag, New York, Heidelberg, Berlin, 1978).

[21] K. Hicks (2007), hep-ph/0703004.

[22] R. De Vita et al. (CLAS), Phys. Rev. D74, 032001 (2006), hep-ex/0606062.

[23] F. Csikor, Z. Fodor, S. D. Katz, T. G. Kovacs, and B. C. Toth, Phys. Rev. D73, 034506 (2006), hep-lat/0503012.

[24] S. Sasaki, Nucl. Phys. A752, 165 (2005).

[25] N. Mathur et al., Phys. Rev. D70, 074508 (2004), hep$\mathrm{ph} / 0406196$.

[26] Experience has shown [7] that zero-distance operators give rise to discontinuous, non-smooth, behavior of the potential at $\vec{r}=0$.

[27] This decomposition easily generalizes to the case of a different number of operators at source and sink.

[28] We have found that solving the generalized eigenvalue problem, as put forward in [14], does not promote this desirable feature.

[29] In principle jackknife samples can also be used but the amount of effort would be prohibitive. 